\title{
Robust correlators
}

\author{
P. Fridman
}

\begin{abstract}
ASTRON, Oudehoogeveensedijk 4, Dwingeloo, 7991PD, The Netherlands
\end{abstract}
e-mail: pa.fridman@astron.nl

Received 8 March 2009 / Accepted 19 April 2009

\section{ABSTRACT}

\begin{abstract}
Context. Radio frequency interference (RFI) already limits the sensitivity of existing radio telescopes in several frequency bands and may prove to be an even greater obstacle for future generation instruments to overcome.

Aims. I aim to create a structure of radio astronomy correlators which will be statistically stable (robust) in the presence of interference. Methods. A statistical analysis of the mixture of system noise + signal noise + RFI is proposed here which could be incorporated into the block diagram of a correlator. Order and rank statistics are especially useful when calculated in both temporal and frequency domains.

Results. Several new algorithms of robust correlators are proposed and investigated here. Computer simulations and processing of real data demonstrate the efficacy of the proposed algorithms.
\end{abstract}

Key words. instrumentation: interferometers - methods: data analysis - methods: statistical

\section{Introduction}

Correlators are central to the signal processing system of radio interferometer, (Thompson et al. 2001). Signals received from radio sources mixed with system noise (sky noise + receiver noise) can be represented as stochastic processes with a Gaussian (normal) distribution. Each pair of random numbers at the input of the correlator is described as a bivariate random value $(X, Y)$ with the distribution $\mathcal{N}\left(\mu_{X}, \mu_{Y}, \sigma_{X}^{2}, \sigma_{Y}^{2}, \rho_{0}\right)$ where $\mu_{X}=\mu_{Y}=0$ are the mean values, $\sigma_{X}^{2}$ and $\sigma_{Y}^{2}$ are variances proportional to the intensity of the input noise and $\rho$ is the correlation coefficient (normalized visibility for a particular baseline). From the sequences $x_{1}, \ldots, x_{N}$ and $y_{1}, \ldots, y_{N}$ the correlator calculates the so-called Pearson's correlation coefficient

$r_{\mathrm{P}}=\frac{\sum_{i=1}^{n}\left(x_{i}-\bar{X}\right)\left(y_{i}-\bar{Y}\right)}{\sqrt{\sum_{i=1}^{n}\left(x_{i}-\bar{X}\right)^{2}} \sqrt{\sum_{i=1}^{n}\left(y_{i}-\bar{Y}\right)^{2}}}$,

where $\bar{X}$ and $\bar{Y}$ are the arithmetic averages of $x$ and $y$, respectively. The value $\lim _{n \rightarrow \infty} r_{\mathrm{P}}=\rho_{0}$. This is valid both for XF and FX correlators (the correlation of complex numbers is reduced to separate correlations of the real and imaginary components).

The sample correlation coefficient $r_{\mathrm{P}}$ is very sensitive to outliers (samples which are not consistent with the normal distribution $\mathcal{N}\left(\mu_{X}, \mu_{Y}, \sigma_{X}^{2}, \sigma_{Y}^{2}, \rho_{0}\right)$, i.e., the estimate (1) is not statistically robust (Gnanadesikan 1997; Huber 1981). Radio frequency interference (RFI) can produce considerable outliers, yielding a bias of $r_{\mathrm{P}}$ and increasing the standard deviation (rms) of $r_{\mathrm{P}}$. In this paper the terms "RFI" and "outliers" are interchangeable.

Methods of robust statistics allow stable estimates to be obtained in the presence of outliers in several radio-astronomical applications (Fridman 2008). Here these methods are applied to correlation measurements of radio interferometers.

There are different types of RFI (Fridman \& Baan 2001) and they differ significantly over the radio astronomy frequency band. Strong and impulse-like RFI on the time-frequency plane are visible in practically all frequency bands of LOFAR, and

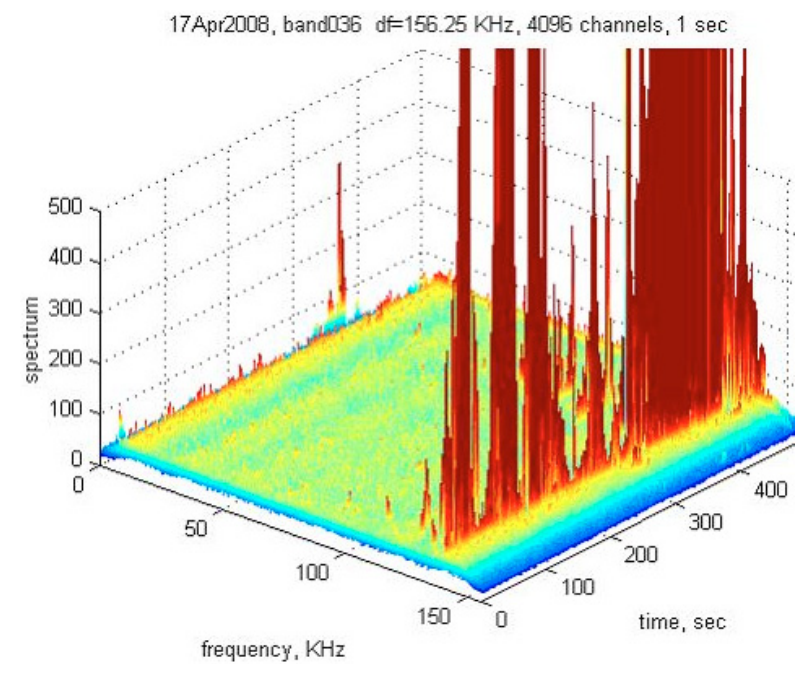

Fig. 1. Autospectrum of system noise + RFI from LOFAR CS1 at the central frequency $f_{0}=217.1875 \mathrm{MHz}$, bandwidth $\Delta f=156.25 \mathrm{KHz}$, frequency resolution $\delta f \approx 38 \mathrm{~Hz}$, integration time $t \approx 1 \mathrm{~s}$.

these types of RFI are considered here because data from the LOFAR Core Station (CS1) are used as examples later in this paper. Figure 1 gives an impression of the types of interferences in one sub-band: the auto-spectrum of the system noise + RFI from the LOFAR CS1 is represented in this figure with high spectral and time resolution.

The following approximate classification is proposed for the strong RFI visible at LOFAR:

a) narrow-band and persistent RFI;

b) narrow impulses $(<1 \mathrm{~s},<100 \mathrm{~Hz})$ on the time-frequency plane;

c) impulse-like RFI in the time domain (several seconds) which are also wide-band (5-10 MHz);

d) random-form bursts on the time-frequency plane. 
There are several types of RFI which cannot be treated as outliers: they may be persistent and wide-band, weak or strong, fully or partly correlated at the sites of a radio interferometer. Methods using the spatial separation of the source of RFI and the radio source can effectively address this issue, see (Ellingson \& Hampson 2003; Jeff et al. 2005; Kesteven 2007; Cornwell et al. 2004).

Several types of robust correlators that are able to mitigate the impulse-like, strong RFI in both temporal and frequency domains have been studied in this paper. They are used in applications where input data are contaminated with outliers, and these correlators are statistically more stable than correlator (1). Some of them could be used in radio astronomy, especially in radio interferometric systems with software correlators where the calculation of visibilities is carried out on general-purpose computers, as in LOFAR (2009) and JIVE (Kruithof2009). Software correlators are, by definition, much more flexible than traditional hardware correlators: any algorithm adapted or modified for a particular observation can be optionally downloaded as a subroutine.

There are two operations in the numerator of (1): multiplication of the input samples of $X$ and $Y$ and summation (averaging). Here it is proposed that they be modified to make the correlator more robust.

The new features can be introduced in the first operation to analyze the statistics of $X$ and $Y$ and to introduce a type of editing in order to eliminate outliers.

The second operation, summation, which is considered as post-correlation averaging, can be divided into three steps: primary averaging over a time interval which is not too long to smooth RFI bursts appearing at this stage above the noise level, RFI mitigation and secondary averaging to the required time interval depending on the observational specifications (wavelength, baseline, radio source properties), see end of Sect. 3 and Figs. 13-15.

Different types of correlators described in the following section are compared with Pearson's correlator (1) using two criteria:

1. the bias of the estimate $\widehat{\rho}$ produced by RFI compared to the input correlation coefficient $\rho_{0}$;

2. the effectiveness of an estimator is judged by the rms at the output of the correlator in both the presence and in the absence of outliers.

Computer simulations were performed to estimate these values, of the bias and rms. Also some results of the processing of CS1 data will be shown.

\section{Estimators of the correlation coefficient}

There are two classic estimators of correlation coefficients using ranks of samples instead of the samples themselves, (Kendall 1970).

Let two input sample sequences $x_{1}, \ldots x_{n}$ and $y_{1}, \ldots y_{n}$ be sorted in ascending order: $x_{(1)} \leq x_{(2)} \leq \ldots \leq x_{(n)}$ and $y_{(1)} \leq y_{(2)} \leq$ $\ldots \leq y_{(n)}$. The ith value $x_{(i)}$ is called ith-order statistic. Each sample $x_{j}$ has its $k$ th position in the sorted series $x_{(1)}, \ldots x_{(n)}$. This number $k$ is the rank of the sample $x_{j}$ and is denoted by $p_{j}(=k)$. Similarly, the rank of $y_{j}$ is denoted by $q_{j}$. Let $\left(x_{i}, y_{i}\right)$ and $\left(x_{j}, y_{j}\right)$ with $i=1, \ldots n$ and $j=i+1, \ldots n$ be two data pairs from the original data sequences. If $p_{j}-p_{i}$ and $q_{j}-q_{i}$ have the same sign, these two data pairs are said to be concordant, otherwise, they are discordant. Let $n_{\mathrm{c}}$ be the number of concordant pairs and $n_{\mathrm{d}}$ the number of discordant pairs. It is clear that $n_{\mathrm{c}}+n_{\mathrm{d}}=n(n-1) / 2$.

\subsection{Spearman's correlator}

The Spearman's correlation coefficient is calculated by

$r_{\mathrm{SP}}=1-\frac{6 \sum_{i=1}^{n}\left(p_{i}-q_{i}\right)^{2}}{n\left(n^{2}-1\right)}$

The bivariate correlation coefficient corresponding to Pearson's $r_{\mathrm{P}}$ can be restored using the relationship:

$\widehat{\rho}=2 \sin \left(\frac{1}{6} \pi r_{\mathrm{SP}}\right)$

\subsection{Kendall's correlator}

Kendall's correlation coefficient is calculated by

$r_{\mathrm{KND}}=\frac{2\left(n_{\mathrm{c}}-n_{\mathrm{d}}\right)}{n(n-1)}$.

The bivariate correlation coefficient corresponding to Pearson's $r_{\mathrm{P}}$ can be restored using the relationship:

$\widehat{\rho}=\sin \left(\frac{1}{2} \pi r_{\mathrm{KND}}\right)$.

\subsection{Correlator using sums and differences}

One of the first constructions of a robust correlator is based on the quarter square identity (Gnanadesikan \& Kettenring 1972; Huber 1981):

$\operatorname{cov}(X, Y)=1 / 4[\operatorname{var}(X+Y)-\operatorname{var}(X-Y)]$,

where cov denotes covariance and var denotes variance. The correlation coefficient can be obtained with

$r_{\mathrm{GK}}=1 / 4 \frac{\operatorname{var}(X+Y)-\operatorname{var}(X-Y)}{\sqrt{\operatorname{var}(X) \operatorname{var}(Y)}}$.

Therefore, any robust estimators of variance can be used for this correlator (Fridman 2008). Several of them are applied here to (7).

\subsubsection{Trimming}

Samples $Z_{1}=X+Y$ and $Z_{2}=X-Y$ are sorted in ascending order: $z_{(1)}, \ldots z_{(n)}$. Let $\gamma$ denote the chosen amount of trimming, $0 \leq \gamma \leq 0.5$ and $k=[\gamma n]$. Sample-trimmed variance is computed by removing $k$ of the largest and $k$ of the smallest data and using the values that remain:

$$
\begin{aligned}
\operatorname{var}_{\text {trim }} & =\frac{K_{\text {trim }}}{n-2 k} \sum_{i=k}^{n-k}\left(z_{i}-\widehat{\mu}_{\text {trim }}\right)^{2} \\
\widehat{\mu}_{\text {trim }} & =\frac{1}{n-2 k} \sum_{i=k}^{n-k} z_{i},
\end{aligned}
$$

where $\mu_{\text {trim }}$ is the sample mean of the trimmed data. Trimming lessens the variance of data and the coefficient $K_{\text {trim }}$ makes var trim a consistent estimator for data with a normal distribution. The value of $K_{\text {trim }}$ depends on $\gamma$, (Fridman 2008), but in the context of Eq. (7), this is not important, because $K_{\text {trim }}$ appears symmetrically in the numerator and denominator of (7). 


\subsubsection{Winsorization}

A sample $Z$ is sorted in ascending order. For the chosen $0 \leq \gamma \leq$ 0.5 and $k=[\gamma n]$, Winsorization of the sorted data consists of setting

$W_{i}= \begin{cases}z_{(k+1)}, & \text { if } z_{(i)} \leq z_{(k+1)} \\ z_{(i)}, & \text { if } z_{(k+1)}<z_{(i)}<z_{(n-k)} \\ z_{(n-k)}, & \text { if } z_{(i)} \geq z_{(n-k)} .\end{cases}$

The Winsorized sample mean is $\widehat{\mu_{\mathrm{w}}}=\frac{1}{n} \sum_{i=1}^{n} W_{i}$ and the Winsorized sample variance is

$\operatorname{var}_{\text {wins }}=\frac{1}{n-1} \sum_{i=1}^{n}\left(W_{i}-\widehat{\mu_{w}}\right)^{2}$.

The essence of Winsorization is to replace the $k$ of the lowest and $k$ of the highest samples of the sorted data $z$ by the values of their nearest neighbors $z_{(k+1)}$ and $z_{(n-k)}$, respectively.

\subsubsection{Median absolute deviation (MAD)}

This estimate of the variance of sorted data $z_{(1)} \leq z_{(2)} \leq \ldots \leq z_{(n)}$ is defined by

$\operatorname{var}_{\text {med }}=\left(1.483 \times \operatorname{med}_{1 \leq i \leq n}\left\{\left|z_{i}-\operatorname{med}\left(z_{i}\right)\right|\right\}\right)^{2}$,

where

$$
\begin{array}{ll}
\text { med }=0.5\left(z_{(m)}+z_{(m+1)}\right), & n=2 m, \\
\text { med }=z_{(m+1)}, & n=2 m+1 .
\end{array}
$$

Using $\operatorname{med}\left(z_{i}\right)$ in the place of sample mean and (med $\left.\left|z_{i}-\operatorname{med}\left(z_{i}\right)\right|\right)^{2}$ in the place of sample variance provides a more robust estimate of variance: only central samples of sorted data (central order statistics) are used, according to the definition of the median, and outliers are excluded. This procedure works well with data contaminated by up to $\approx 50 \%$ outliers, but the rms of the estimator (11) is larger than that of the classic variance estimate (see Sect. 3, Table 4).

\subsubsection{Qn estimate}

This estimate is proposed in Rousseuw \& Croux (1993) and is moderately effective. It combines the ideas of the Hodges-Lehmann estimate and the Gini estimate, (Kendal \& Stuart 1967):

$\operatorname{var}_{Q n}=2.108\left\{\left|x_{i}-x_{j}\right|, i<j\right\}_{(k)}$,

where $k=\left(\begin{array}{l}h \\ 2\end{array}\right)$ and $h=[n / 2]+1$, that is $k \approx\left(\begin{array}{l}n \\ 2\end{array}\right) / 4$. This estimator works as follows: the interpoint (pairwise) distances $\left|x_{i}-x_{j}\right|, i<j$, are sorted in ascending order. The $k$ th value of this sorted sequence (the kth order statistics) multipled by consistency factor 2.108 is then taken as the estimate of variance. The value of the consistency factor is also not critical, as for trimming (Sect. 2.3.1).

Other robust algorithms can also be applied for example, the M-estimator, (Huber 1981) but here I was not attempting to make a comprehensive study of all types of robust correlators. Rather I wished to direct attention to the options hitherto unused in designing correlators.
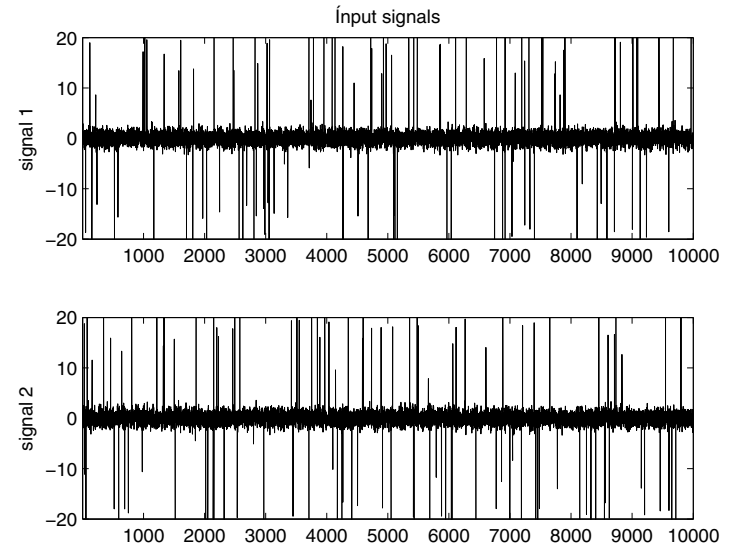

Fig. 2. Input signals representing Gaussian noise + impulse-like outliers, the number of samples $n=10000$.

\section{Testing the algorithms}

\subsection{Mitigation of outliers in the temporal domain}

The two input signals $X$ and $Y$ are modeled by bivariate random numbers with normal distribution, zero mean, standard deviation $\sigma=1.0$ and correlation coefficient $\rho=0.0 ; 0.05 ; 0.1 ; 0.2$. Such $\rho$ are typical of many radio interferometric observations when weak signals are processed. The number of samples in each data sequence is $n$ and the number of repetitions of the correlation is $m$. Outliers $r f i_{i}$ added to the inputs $x_{i}$ and $y_{i}$ are modeled by the following expression:

$r f i_{i}=s_{i} \times \operatorname{sign}\left(z_{i}\right) \times\left(A_{\mathrm{rfi}}+\sigma_{\mathrm{rfi}} \times u_{i}\right)$

where $s_{i}$ is the Poisson random value with the parameter $\lambda=$ $0.01, A_{\mathrm{rfi}}=20.0$ is the amplitude of outliers randomized by adding the normal random numbers $u_{i}$ with the standard deviation $\sigma_{\text {rfi }}=0.3 A_{\text {rfi }}$. The random polarities of the outliers are provided by the $\operatorname{sign}\left(z_{i}\right), z_{i}$ is the other auxiliary random normal number. Figure 2 gives the example of a sequence of input samples.

The results of computer simulations, bias and rms of the estimate of $\widehat{\rho}$ are presented in Tables $1-5$. The bias of the estimate $\widehat{\rho}$ is: bias $=\widehat{\rho}-\rho$. The confidence intervals are calculated for the $95 \%$-confidence level and for $\operatorname{rms}_{\widehat{\rho}}=1 / \sqrt{m n}$ for $\widehat{\rho}$ and $\mathrm{rms}_{\widehat{\mathrm{rms}}}=1 / \sqrt{2 m n}$ for rms (the approximations that are valid for small $\rho$ and large $m n$ ).

Looking at Tables 1-5 several remarks can be made:

1. in the absence of outliers, robust correlators reproduce practically the same values of $\widehat{\rho}$ as the Pearson's correlator (not showing a significant bias) and keeping the effectiveness close to $1 / \sqrt{m n}$ (except MAD). Figure 3 shows this for correlator (7) with Winsorization;

2. the output of the Pearson's correlator is dramaticaly decorrelated with the growth of the outlier's amplitudes whereas robust correlators analyze local statistics ( $n$ samples) and eliminate outliers regardless of their amplitudes, see Fig. 4;

3 . in the presence of outliers, robust correlators show a small positive bias which is equal to $\approx 1.0 \%$ of $\rho$;

4. Spearman's and Kendall's correlators (Table 1) provide a considerable reduction of bias in the presence of RFI compared to the reduction of bias in Pearson's correlator and keep the rms, i.e., there is no loss in effectiveness within the 
Table 1. Bias and rms of $\widehat{\rho}$ for the Spearman and Kendall correlators, $n=1000, m=1000,95 \%$-confidence intervals for bias: $\delta_{\text {bias }}= \pm 0.00205$, for rms: $\delta_{\mathrm{rms}}= \pm 0.0014$.

\begin{tabular}{|c|c|c|c|c|c|c|}
\hline \multicolumn{7}{|c|}{ Without outliers } \\
\hline & \multicolumn{2}{|c|}{ Pearson } & \multicolumn{2}{|c|}{ Spearman } & \multicolumn{2}{|c|}{ Kendall } \\
\hline$\rho$ & Bias & Rms & Bias & Rms & Bias & Rms \\
\hline 0.00 & 0.000013 & 0.03265 & 0.000101 & 0.03425 & 0.000100 & 0.03428 \\
\hline 0.05 & -0.000672 & 0.03245 & -0.000436 & 0.03391 & -0.000366 & 0.03396 \\
\hline 0.10 & 0.000075 & 0.03268 & 0.000431 & 0.03454 & 0.000511 & 0.03452 \\
\hline 0.20 & 0.000445 & 0.03023 & 0.000696 & 0.03217 & 0.000817 & 0.03207 \\
\hline \multicolumn{7}{|c|}{ Without outliers } \\
\hline & \multicolumn{2}{|c|}{ Pearson } & \multicolumn{2}{|c|}{ Spearman } & \multicolumn{2}{|c|}{ Kendall } \\
\hline$\rho$ & Bias & Rms & Bias & Rms & Bias & Rms \\
\hline 0.00 & 0.00156 & 0.03075 & 0.000416 & 0.03453 & 0.000419 & 0.03455 \\
\hline 0.05 & -0.04115 & 0.03026 & -0.000179 & 0.03399 & -0.000131 & 0.03405 \\
\hline 0.10 & -0.07844 & 0.03212 & -0.003240 & 0.03393 & -0.003159 & 0.03392 \\
\hline 0.20 & -0.16020 & 0.03144 & -0.007427 & 0.03233 & -0.007243 & 0.03222 \\
\hline
\end{tabular}

Table 2. Bias and rms of $\widehat{\rho}$ for trimming, $\gamma=0.1, n=1000, m=10000,95 \%$-confidence intervals for bias: $\delta_{\text {bias }}= \pm 0.00062$, for rms: $\delta_{\text {rms }}=$ \pm 0.00044 .

\begin{tabular}{ccccccccc}
\hline \hline & \multicolumn{4}{c}{ Without outliers } & \multicolumn{3}{c}{ With outliers } \\
\hline \multicolumn{2}{c}{ Pearson } & \multicolumn{2}{c}{ Trimming } & \multicolumn{2}{c}{ Pearson } & \multicolumn{2}{c}{ Trimming } \\
$\rho$ & Bias & Rms & Bias & Rms & Bias & Rms & Bias & Rms \\
\hline 0.00 & -0.000176 & 0.031209 & -0.000171 & 0.041631 & -0.000048 & 0.030746 & -0.000193 & 0.042383 \\
0.05 & -0.000398 & 0.031813 & -0.000975 & 0.042481 & -0.039886 & 0.031722 & 0.000503 & 0.043066 \\
0.10 & 0.000326 & 0.031324 & 0.000575 & 0.041588 & -0.079740 & 0.031434 & -0.000943 & 0.042246 \\
0.20 & -0.000556 & 0.030111 & 0.000614 & 0.040910 & -0.160044 & 0.032241 & -0.002102 & 0.041670 \\
\hline
\end{tabular}

Table 3. Bias and rms of $\widehat{\rho}$ for Winsorization, $\gamma=0.1, n=1000, m=10000,95 \%$-confidence intervals for bias: $\delta_{\text {bias }}= \pm 0.00062$, for rms: $\delta_{\text {rms }}= \pm 0.00044$.

\begin{tabular}{ccccccccc}
\hline \hline & \multicolumn{4}{c}{ Without outliers } & \multicolumn{3}{c}{ With outliers } \\
\hline \multicolumn{2}{c}{ Pearson } & \multicolumn{2}{c}{ Winsorization } & \multicolumn{2}{c}{ Pearson } & \multicolumn{2}{c}{ Winsorization } \\
$\rho$ & Bias & Rms & Bias & Rms & Bias & Rms & Bias & Rms \\
\hline 0.00 & 0.000032 & 0.031597 & 0.000046 & 0.037387 & -0.000692 & 0.032661 & 0.000086 & 0.038446 \\
0.05 & -0.000025 & 0.031979 & -0.000105 & 0.037754 & -0.040128 & 0.032028 & -0.000886 & 0.038748 \\
0.10 & -0.000151 & 0.031276 & -0.000396 & 0.037174 & -0.080514 & 0.032396 & -0.002059 & 0.038072 \\
0.20 & 0.000010 & 0.030453 & -0.000253 & 0.036746 & -0.160392 & 0.032851 & -0.002856 & 0.037700 \\
\hline
\end{tabular}

limits of error. These correlators require more operating capacity per lag, due to the sorting and ranking of samples (this is also valid for the following algorithms);

5. trimming and Winsorization (Tables 2 and 3) show good results with regard to bias and effectiveness. The rms for trimming is increased $\approx 10 \%$ than for Winsorization. The choice of $\gamma=0.1$ presumes a percentage of outliers of less than $10 \%$. So there is a considerable safety margin here but the growth of rms for $\gamma=0.1$ is insignificant. In practice, an adaptive choice of $\gamma$ is possible in each sub-band when the value of $\gamma$ is chosen after taking into account a real RFI environment situation, i.e., the presence or absence of RFI and its duty cycle;

6. median absolute deviation (MAD) (Table 4) gives the best results for the bias, but as predicted theoretically, it has the largest rms: $\approx 1.65$ greater than $1 / \sqrt{m n}$;

7. the Qn estimate (Table 5) requires more operating capacity than the others. The bias is as little as for the other algorithms, the rms is slightly larger $(\approx 10 \%)$ than $1 / \sqrt{m n}$;

8. the proposed methods are effective in the presence of strong impulse-like outliers. When not intercepted, they decorrelate the correlator's output and it is practically impossible to improve this situation with subsequent processing. Low-amplitude outliers are more difficult to detect because they are similar to the rare Gaussian noise spikes, but their decorrelation impact is also much weaker. For example, with an RFI amplitude $\approx 3.0 \sigma, \lambda=0.01$ and $\rho=0.1$ (signal-ofinterest), the output of Pearson's correlator is 0.092 and the output of Spearman's correlator 0.098;

9. the situation is different when outliers are correlated at the inputs of the correlator. In this case outliers produce an excessive, false correlation even in the absence of a signal-ofinterest, $\rho=0.0$. For example, for strong $100 \%$-correlated RFI with an amplitude equal to $30.0 \sigma, \lambda=0.001$ and $\rho=0.0$, the output of Pearson's correlator is 0.349 and the output of the MAD-correlator is 0.001 .

For correlated RFI, other methods exploiting this correlation property can be more effective, see (Ellingson \& Hampson 2003; Jeff et al. 2005; Kesteven 2007; Cornwell et al. 2004).

\subsection{Mitigation of outliers in the frequency domain}

Narrow-band RFI can be detected as an impulse-like outlier in the frequency domain. The following example illustrates the application of trimming (8) in this case. RFI is generated as a phase-modulated sinusoidal carrier, see Fig. 5 . The power spectrum of the unmodulated carrier is shown in the upper panel and 
Table 4. Bias and rms of $\widehat{\rho}$ for median absolute deviation (MAD), $n=1000, m=10000,95 \%$-confidence intervals for bias: $\delta_{\text {bias }}= \pm 0.00097$, for rms: $\delta_{\text {rms }}= \pm 0.00069$.

\begin{tabular}{cccccccccc}
\hline \hline & \multicolumn{4}{c}{ Without outliers } & \multicolumn{4}{c}{ With outliers } \\
\hline \multicolumn{4}{c}{ Pearson } & \multicolumn{2}{c}{ MAD } & \multicolumn{3}{c}{ Pearson } & \multicolumn{3}{c}{ MAD } \\
\multicolumn{2}{c}{ Pearson } & \multicolumn{2}{c}{ MAD } & \multicolumn{2}{c}{ Pearson } & MAD \\
$\rho$ & Bias & Rms & Bias & Rms & Bias & Rms & Bias & Rms \\
\hline 0.00 & 0.000073 & 0.031411 & -0.000057 & 0.051588 & 0.000246 & 0.031372 & -0.000097 & 0.052656 \\
0.05 & 0.000073 & 0.031761 & -0.000315 & 0.052502 & -0.039889 & 0.032401 & 0.001113 & 0.053639 \\
0.10 & 0.000064 & 0.031470 & -0.000396 & 0.051782 & -0.080238 & 0.031536 & 0.002077 & 0.052777 \\
0.20 & -0.000510 & 0.030379 & -0.000158 & 0.051560 & -0.080546 & 0.033214 & 0.002244 & 0.052823 \\
\hline
\end{tabular}

Table 5. Bias and rms of $\widehat{\rho}$ for $Q 2$-estimator, $n=1000, m=1000,95 \%$-confidence intervals for bias: $\delta_{\text {bias }}= \pm 0.00205$, for rms: $\delta_{\text {rms }}= \pm 0.0014$.

\begin{tabular}{ccccccccc}
\hline \hline & \multicolumn{4}{c}{ Without outliers } & \multicolumn{3}{c}{ With outliers } \\
\hline \multicolumn{2}{c}{ Pearson } & \multicolumn{2}{c}{ Qn } & \multicolumn{2}{c}{ Pearson } & \multicolumn{2}{c}{ Qn } \\
$\rho$ & Bias & Rms & Bias & Rms & Bias & Rms & Bias & Rms \\
\hline 0.00 & 0.000488 & 0.031669 & 0.000541 & 0.035316 & -0.000122 & 0.030729 & 0.000446 & 0.037311 \\
0.05 & 0.001529 & 0.031255 & 0.001392 & 0.033659 & -0.039906 & 0.032105 & 0.000570 & 0.035250 \\
0.10 & 0.000362 & 0.031255 & 0.000277 & 0.034868 & -0.081281 & 0.031905 & 0.001994 & 0.037097 \\
0.20 & -0.001444 & 0.029626 & -0.001721 & 0.032624 & -0.158926 & 0.033120 & 0.000594 & 0.034818 \\
\hline
\end{tabular}
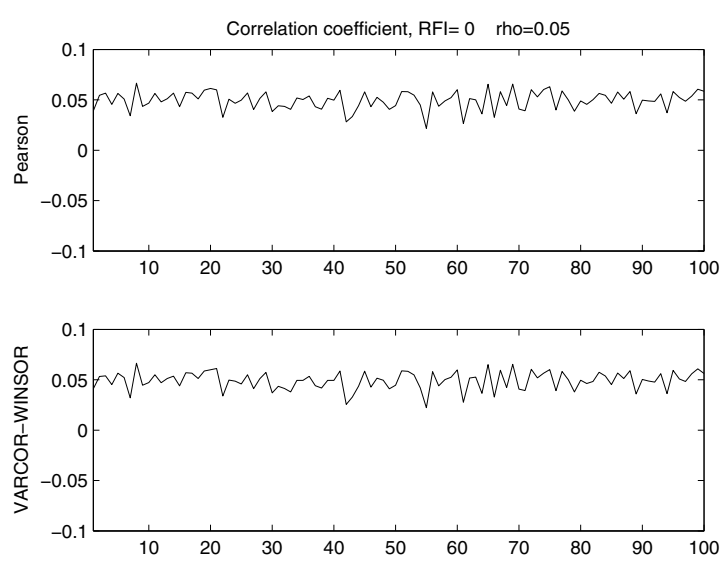

Fig. 3. Correlation coefficients in the absence of outliers for Pearson's correlator (1), (upper panel), and Winsorized correlator (7), (lower panel), each of the $m=100$ correlation coefficients are calculated for $n=1000$ data samples, $\rho=0.05$.

in the lower panel, the power spectrum of the randomly phasemodulated signal is represented:

$s=A_{\mathrm{rfi}} \sin (2 \pi F i+(\pi / 2) \operatorname{rect}(i)), i=1 \ldots n$

where $\operatorname{rect}(i)$ is a rectangular wave with Poisson's law distributed random jumps from -1 to +1 , and the parameter of Poisson distribution $\lambda=0.01$. The mixture of two input signals and their power spectra are represented in Fig. 6. The amplitudes $A 1_{\text {rfi }}=A 2_{\text {rfi }}=0.5$ and frequencies $F 1=0.3$ and $F 2=0.4$. Therefore, RFI is not visible in the temporal domain but is easily visible in the spectral domain after FFT with $n=2048$ as two bursts.

The statistics of the power spectra are analyzed following (8) and the indexes of outliers exceeding the level equal to the $1.5 \%$-percentile are used to reject the samples of the complex spectra of signals $\widetilde{x}$ and $\widetilde{y}$, where $\widetilde{(.)}$ denotes the Fourier transform. These indexes are tagged with the indicator function ind $(i)$ taking the numbers 0 or $1, i=1 \ldots n$ and assigned to the sequence of $n$ samples of the complex spectra $\widetilde{x}$ and $\widetilde{y}$.
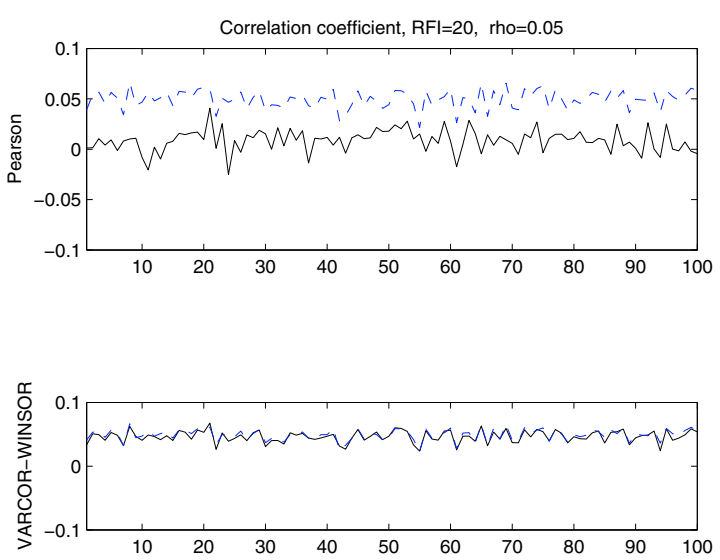

Fig. 4. Correlation coefficients in the presence of outliers for Pearson's correlator (1), (upper panel), and Winsorized correlator (7), (lower panel), $A_{\mathrm{rfi}}=20.0, \rho=0.05$. The solid line in the upper panel shows the total loss of correlation, the dashed line corresponds to the absence of outliers, while the solid and dashed lines in the lower panel practically coincide.
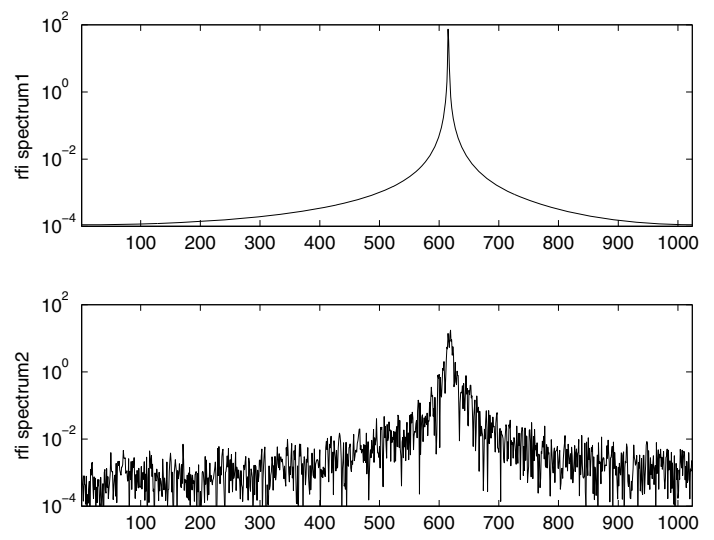

Fig. 5. Power spectra of the unmodulated sinusoidal carrier (upper panel) and the randomly-phased modulated carrier with the phase jumps $\pm \pi / 2$. 


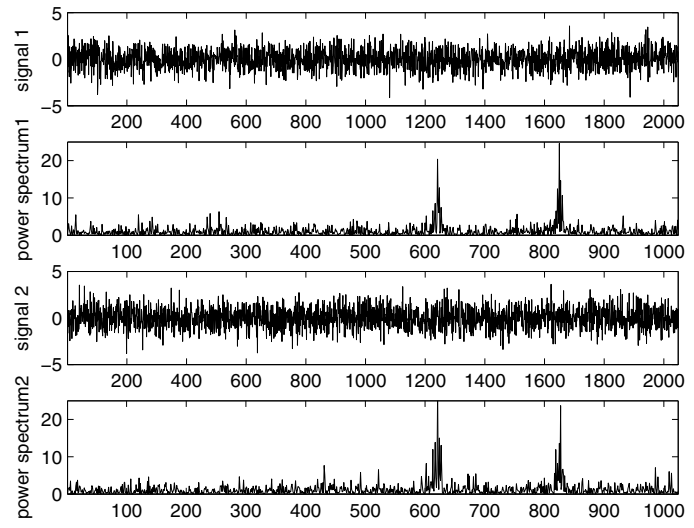

Fig. 6. Input signal $x$ noise + RFI (upper panel) and its power spectra (upper middle panel), input signal $y$ (lower middle panel) and its power spectra (lower panel).
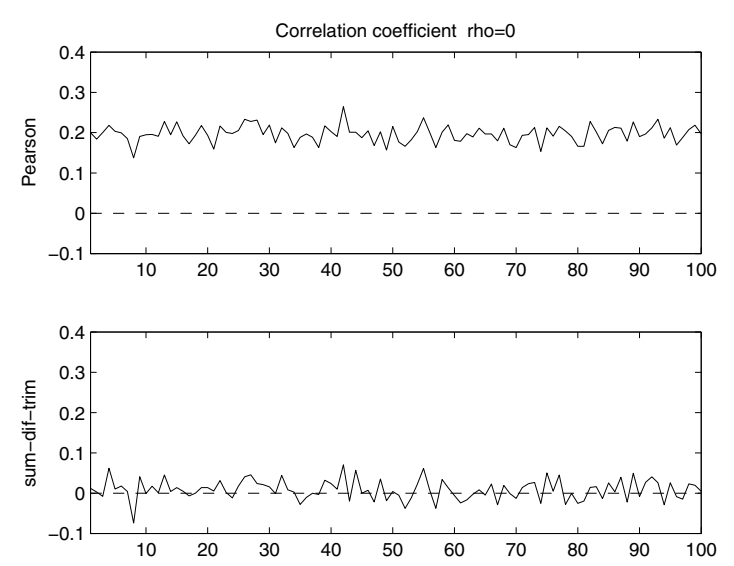

Fig. 7. Pearson's correlator output (upper panel) and the "sumdifference" correlator ouput (lower panel), $\rho=0.0$.

The "sum-difference" correlator (7) is used in the spectral domain (FX-correlator):

$$
\begin{array}{r}
\operatorname{var}(\operatorname{sum})=\operatorname{var}(\mathfrak{R}(\text { sum }))+\operatorname{var}(\mathfrak{J}(\text { sum })), \text { sum }=\widetilde{x}+\widetilde{y} \\
\operatorname{var}(\text { dif })=\operatorname{var}(\mathfrak{R}(\text { dif }))+\operatorname{var}(\mathfrak{J}(\text { dif })), \text { dif }=\widetilde{x}-\widetilde{y} .
\end{array}
$$

Robust variance using the trimming algorithm (8) is applied while calculating (15): outliers are marked by the indicator function ind $(i)$. The results of computer simulation for $n=2048$ and $m=100$ are shown in Figs. 7-9. Figure 7 shows the $m$ outputs of the Pearson's correlator, upper panel, and the robust correlator (15), lower panel, for the correlation coefficient of the input signals $\rho=0$, i.e., for the uncorrelated inputs, except RFI, which are $100 \%$ correlated. The upper panel shows considerable bias, while the fluctuations of correlator output in the lower panel vary around zero. Figure 8 gives the same situation but for $\rho=0.1$ and Fig. 9 - for $\rho=0.2$. In all these examples a considerable bias is visible for the Pearson's correlator and there is an absence of bias for the "sum-difference" correlator.

Of course, other post-correlation methods can be used in the case of narrow-band persistent RFI with a stable spatial orientation, for example, (Cornwell et al. 2004). But in the case of sporadic burst-like RFI, the proposed pre-correlation statistical analysis is more appropriate: only $n \approx 2000$ samples were used for each correlator input, which corresponds to a microsecond time scale for typical bandwidths.
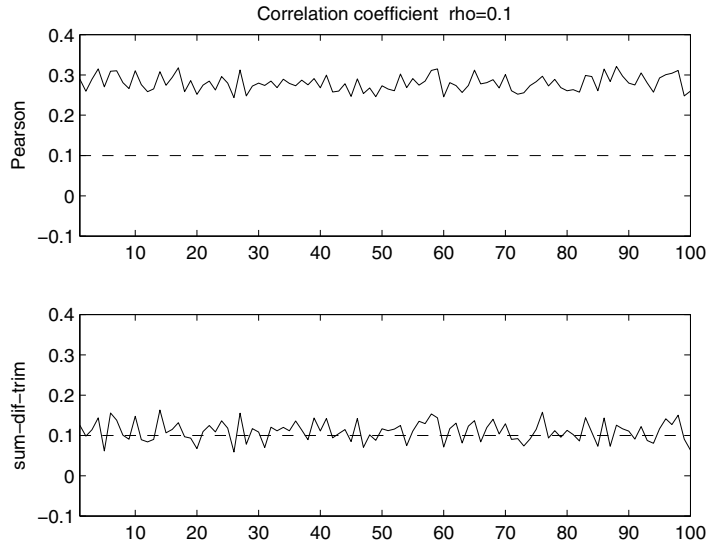

Fig. 8. Pearson's correlator output (upper panel) and the "sumdifference" correlator ouput (lower panel), $\rho=0.1$.
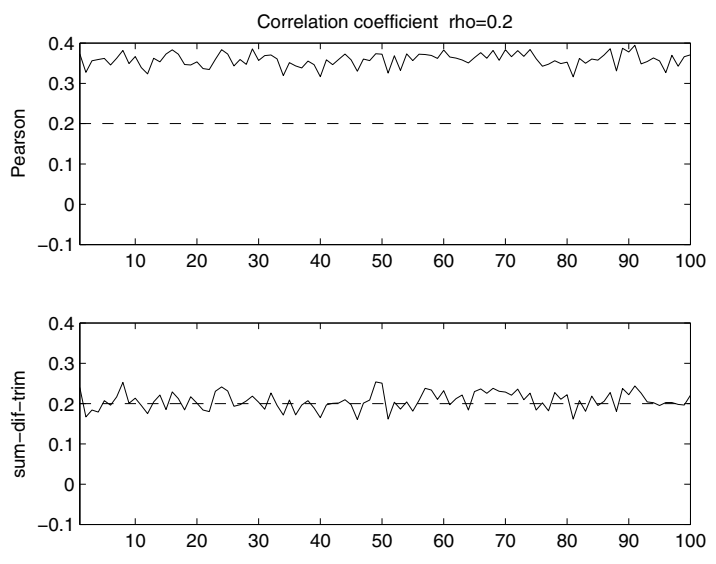

Fig. 9. Pearson's correlator output (upper panel) and the "sumdifference" correlator ouput (lower panel), $\rho=0.2$.

\subsubsection{Processing of observational data}

Several examples of applications of the aforementioned algorithms are presented here.

The auto-spectrum in Fig. 10 is calculated using the "raw" data recorded at LOFAR CS1 for three hours. Data consisting of complex eight-bit numbers and having a sample time interval equal to $6.4 \mu$ s were recorded on the subband with central frequency $f_{0}=205.781 \mathrm{MHz}$, bandwidth $\Delta f=156.25 \mathrm{KHz}$. This time-frequency presentation corresponds to 32 frequency channels, i.e., the frequency resolution is $\delta f \approx 4.88 \mathrm{KHz}$, and the integration time $t \approx 1.7 \mathrm{~s}$.

Figure 11 demonstrates the auto-spectrum calculated from the same data for 1024 frequency channels. The high spectral resolution, $\delta f \approx 153 \mathrm{~Hz}$, permits the separation of RFI spikes, i.e., not smoothing them, as would have been in the case of 32 channels, see Fig. 10. Only half of the 3-h duration autospectrum is shown in Fig. 11 (due to the constraints of computer memory). The auto-spectrum in Fig. 11 is calculated without any censoring of outliers.

Figure 12 shows the "cleaned" auto-spectrum where Winsorization was applied. The Winsorized spectrum in Fig. 12 is smoothed so as to get a final frequency resolution $\delta f \approx$ $4.88 \mathrm{KHz}$ as in Fig. 10. 


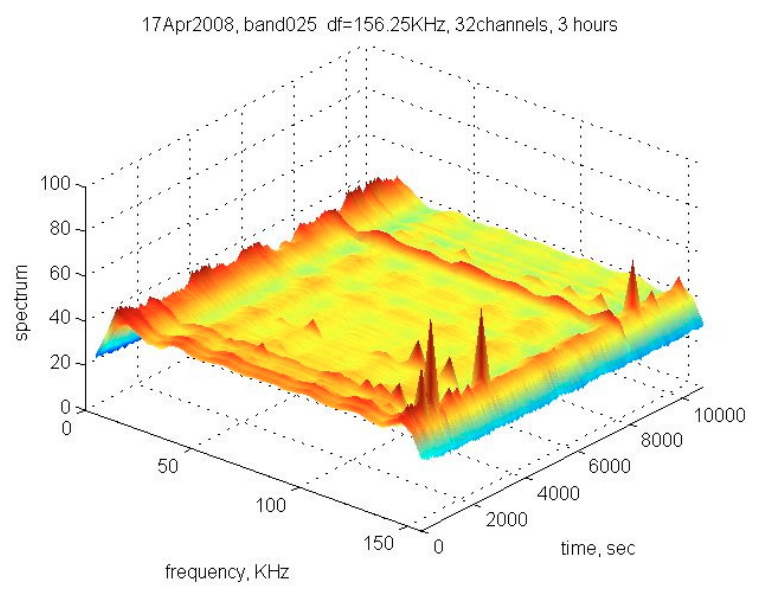

Fig. 10. Auto-spectrum of system noise +RFI from LOFAR CS1 at the central frequency $f_{0}=205.781 \mathrm{MHz}$, bandwidth $\Delta f=156.25 \mathrm{KHz}$, frequency resolution $\delta f \approx 4.88 \mathrm{KHz}$, integration time $t \approx 1.7 \mathrm{~s}$.

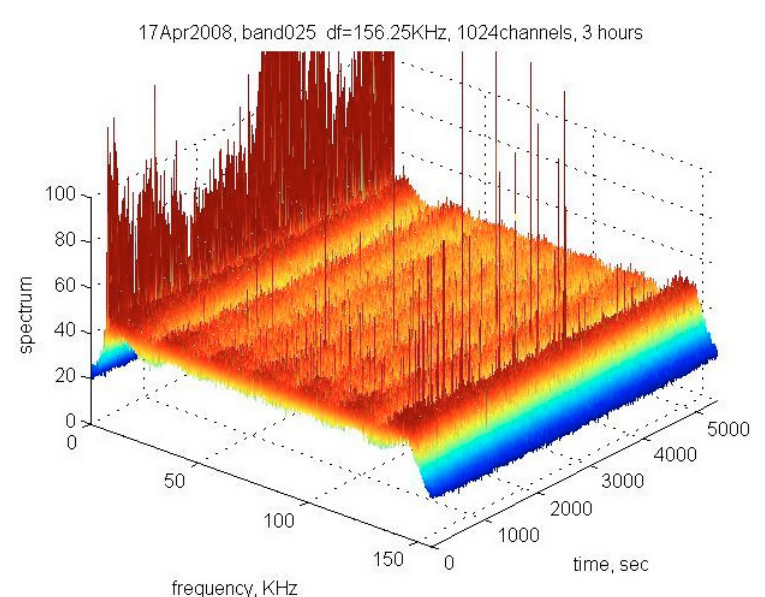

Fig. 11. Auto-spectrum of system noise + RFI from LOFAR CS1 at the central frequency $f_{0}=205.781 \mathrm{MHz}$, bandwidth $\Delta f=156.25 \mathrm{KHz}$, frequency resolution $\delta f \approx 153 \mathrm{~Hz}$, integration time $t \approx 1.7 \mathrm{~s}$.

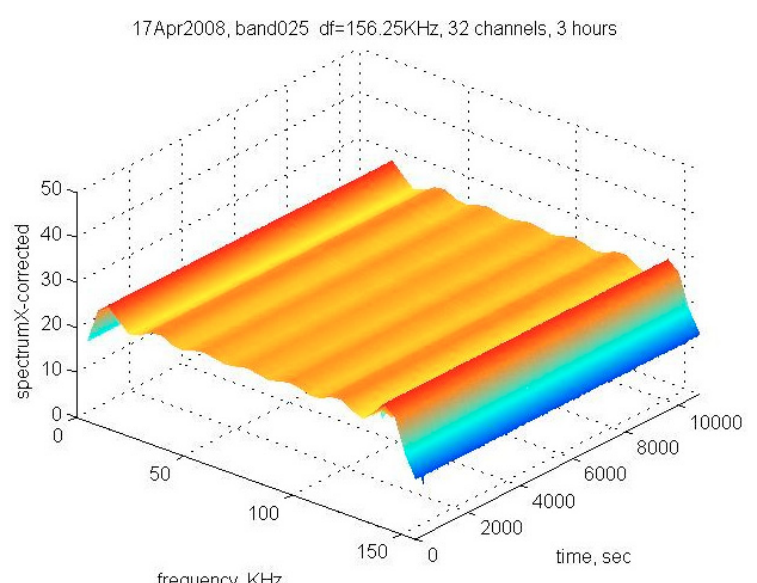

Fig. 12. Auto-spectrum of the same data as in Fig. 11 calculated with Winsorization.

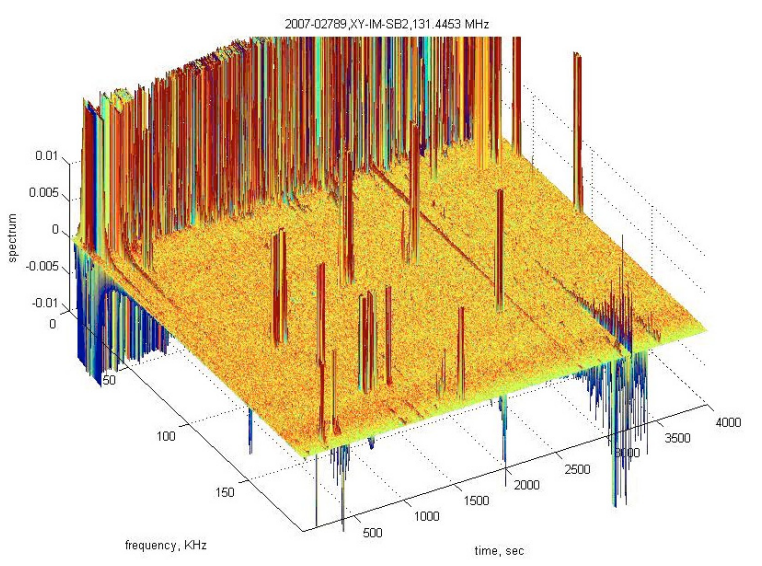

Fig. 13. Post-correlation cross-spectrum, central frequency $f_{0}=$ $131.4453 \mathrm{MHz}$, bandwidth $\Delta f=156.25 \mathrm{KHz}$, frequency resolution $\delta f=610 \mathrm{~Hz}$, time resolution $\delta t=1 \mathrm{~s}$.

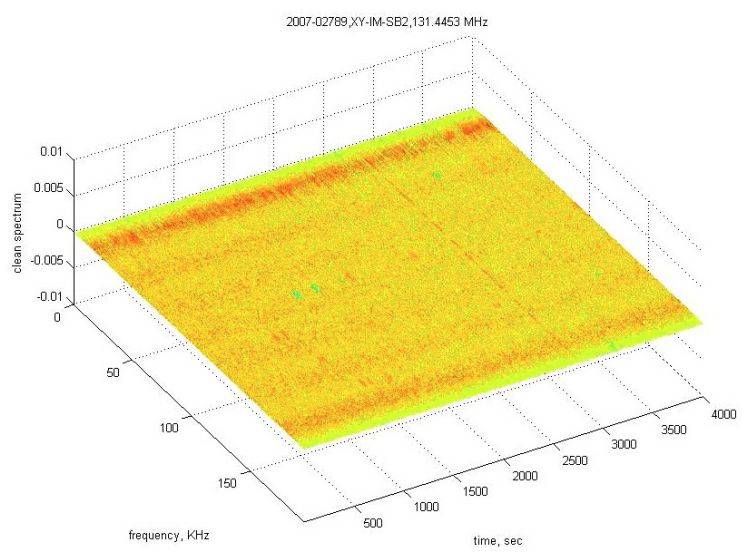

Fig. 14. Post-correlation cross-spectrum from Fig. 13 with outliers removed.
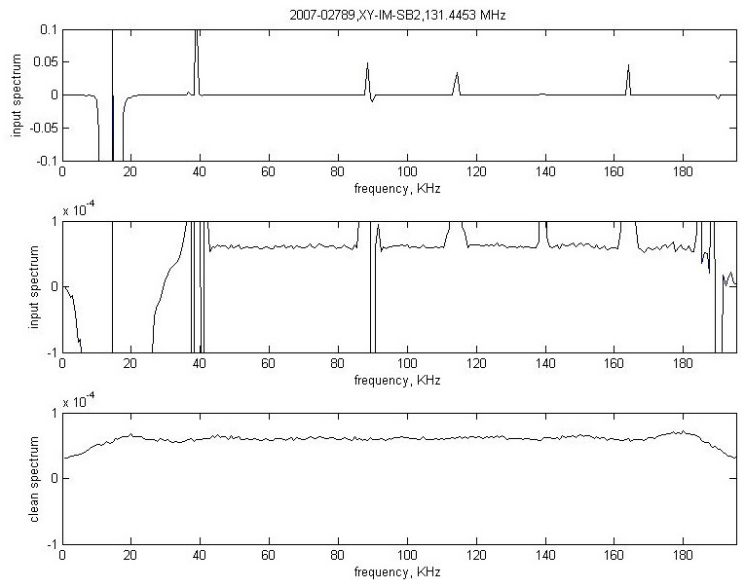

Fig. 15. Averaged cross-spectra corresponding to Fig. 13 (upper and middle (zoomed) panels) and to Fig. 14 (lower panel). 
This example shows the importance of a sufficiently high frequency resolution. All RFI visible in Fig. 11 are invisible in the temporal domain; they are too weak and they are "below" the system noise. The low level of RFI also made it necessary to average the instantaneous power spectra obtained after each FFT. The number of averaged spectra is 256 and thus the time resolution in the Fig. 11 is $6.4 \times 10^{-6} \times 1024 \times 256=1.677 \mathrm{~s}$. The ripple structure clearly visible in the auto-spectra is produced by the transfer functions of LOFAR digital filters separating the whole input bandwidth into dozens of sub-bands with the partial bandwidths $\Delta f=156.25 \mathrm{KHz}$ (or $200 \mathrm{KHz}$ ).

The censoring of outliers may also be useful in the case of post-correlation data produced by the LOFAR correlator. The filter bank of the LOFAR backend divides each of the $156 \mathrm{KHz}$ bandwidth sub-bands into 256 narrow "sub-sub-bands" with a bandwidth equal to $610.3516 \mathrm{~Hz}$. The sample time interval after preliminary averaging is $1 \mathrm{~s}$. This good time-frequency resolution allows the fine-grain structure of RFI to be seen.

The following example in Fig. 13 shows the post-correlation cross-spectrum on frequency $f_{0}=131.4453 \mathrm{MHz}$.

Figure 14 shows the corresponding Winsorized crossspectrum and Fig. 15 shows the averaged cross-spectra along the whole time interval $4 \times 10^{4} \mathrm{~s}-$ without Winsorization (upper and middle panels) and with Winsorization (lower panel). The weak correlated component (the signal-of-interest) is produced by some cross-polarization effects and bears a ripple structure similar to the auto-spectra.

\section{Conclusions}

1. Estimates of the correlation coefficient are an important part of both classical and of robust statistics. Statistical analysis of raw data with the finest available time and frequency resolution can help during observations in an RFI contaminated environment. Growing concern about RFI pollution should persuade the radio astronomy community to pay more attention to the variety of algorithms developed in the realm of robust statistics. This framework of robust estimates puts the successfully tested blanking of RFI on a more stable foundation.
2. Statistically faithful, robust estimates of the correlation coefficient are especially appropriate for application in an impulse-like strong RFI environment. RFI is effectively suppressed and the accompanying bias and effectiveness are tolerable. The aforementioned robust algorithms can be usefully applied in these particular situations.

3. The choice of a particular algorithm depends upon the type and intensity of the RFI. The proportion of RFI presence in data is also important. The type of implementation may determine the choice: off-line or real-time. It is difficult to equip existing conventional hardware correlators with these tools. Future generations of radio telescopes (LOFAR, ATA, SKA) will generate such huge amounts of data that real-time processing is vital: DSP, FPGA or supercomputers are possible solutions. Software correlators are especially suited to the implementation of robust schemes as optional subroutines.

Acknowledgements. My discussions with Ger de Bruyn and Jan Noordam have been very helpful and I am grateful for their continued support.

\section{References}

Cornwell, T. J., Perley, R. A., Golap, K., \& Bhatnagar, S. 2004, EVLA Memo 86 Devlin, S. J., Gnanadesikan, R., \& Kettenring, J. R. 1975, Biometrica, 62, 531 Ellingson, S. W., \& Hampson, G. A. 2003, ApJS, 147, 167

Fridman, P. A. 2008 AJ, 135, 1810

Fridman, P. A., \& Baan, W. 2001, A\&A, 378, 327

Gnanadesikan, R., \& Kettenring, J. R. 1972, Biometrics, 28, 81

Gnanadesikan, R. 1997, Methods for Statistical Data Analysis of Multivariate Observations (John Wiley \& Sons, Inc.), Chap. 5.2.3

Jeff, B. D., Li, L., \& Warnick, K. 2005, IEEE Trans. Signal Process., 53, 439

Huber, P. J. 1981, Robust Statistics (John Wiley \& Sons, Inc.), Chap. 8

Kendall, M. G. 1970, Rank Correlation Methods, 4th edn (London: Griffin)

Kendall, M. G., \& Stuart, A. 1967, The advanced theory of statistics (London: Griffin), 1, Chap. 3

Kesteven, M. 2007, URSI Radio Sci. Bull., 322, 9

Kruithof, N. 2009, Signals and Communication Technology (US: Springer), 537 LOFAR, http://www. lofar.org/p/systems_centrprocess.htm Rousseuw, P. J., \& Croux, C. 1993, J. Amer. Stat. Assoc., 88, 1273

Thompson, A. R., Moran, J. M., \& Swenson, G. W. 2001, Interferometry and Synthesis in Radio Astronomy (John Wiley \& Sons, Inc.), Chap. 8 